\title{
Crystal structure of diethylenetriammonium silver(I) di(4-nitrobenzoate) monohydrate, $\left[\mathrm{Ag}\left(\mathrm{C}_{4} \mathrm{H}_{14} \mathrm{~N}_{3}\right)\right]\left(\mathrm{C}_{7} \mathrm{H}_{4} \mathrm{NO}_{4}\right)_{2} \cdot \mathrm{H}_{2} \mathrm{O}$
}

\author{
H.-L. Zhu*,IIII S.-C. Shao', S. Yang ${ }^{\mathrm{I}}$, X.-Y. Qiu ${ }^{\mathrm{I}}$ J.-L. Ma ${ }^{\mathrm{I}}$ and H.-K. Fun ${ }^{\mathrm{III}}$ \\ I Fuyang Normal College, Department of Chemistry, Fuyang, Anhui, 236041 P. R. China \\ II Wuhan University of Science and Engineering, Department of Chemistry, Wuhan, 430073 P. R. China \\ III Universiti Sains Malaysia, School of Physics, X-Ray Crystallography Unit, 11800 USM, Penang, Malaysia
}

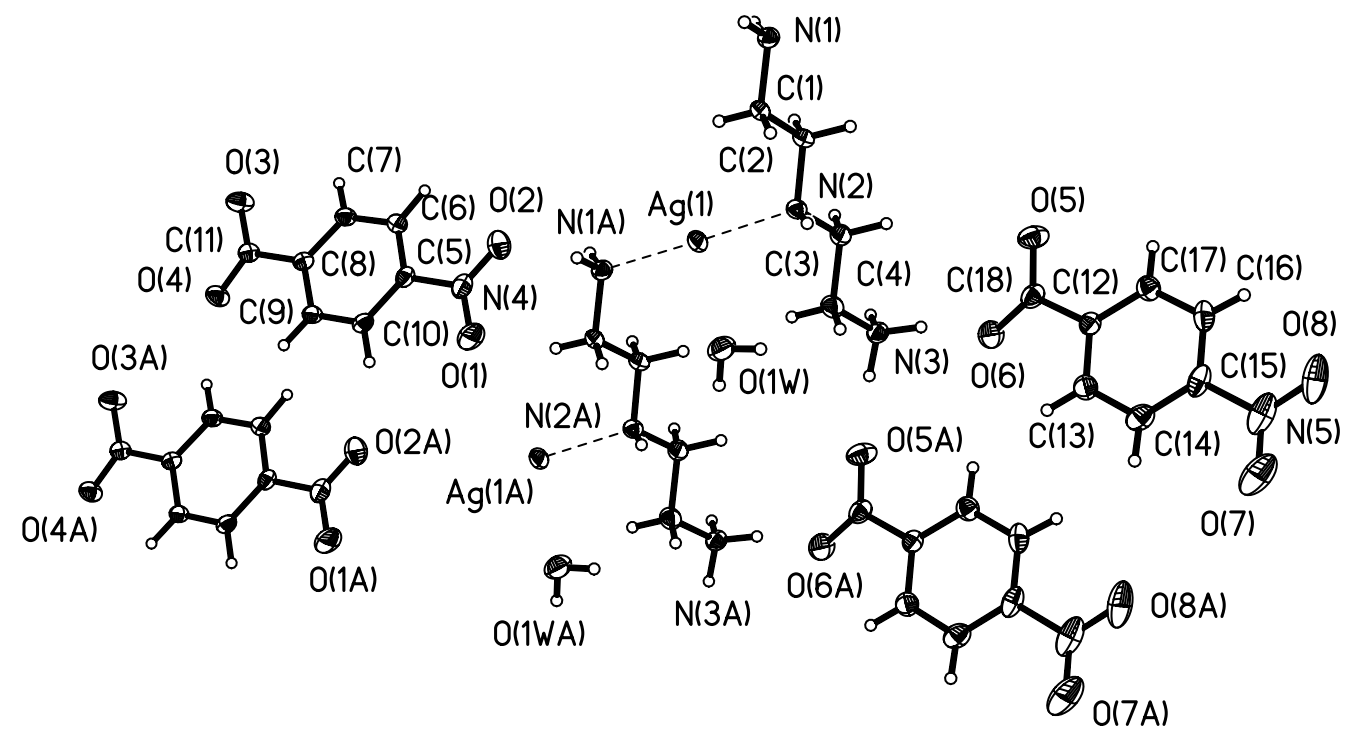

\begin{abstract}
$\mathrm{C}_{18} \mathrm{H}_{24} \mathrm{AgN}_{5} \mathrm{O}_{9}$, monoclinic, $P 121 / n 1$ (No. 14), $a=6.2181(3) \AA$, $b=26.975(2) \AA, c=13.1982(7) \AA, \beta=99.648(2)^{\circ}, V=2182.5 \AA^{3}$, $Z=4, R_{\mathrm{gt}}(F)=0.038, w R_{\mathrm{ref}}\left(F^{2}\right)=0.082, T=293 \mathrm{~K}$.
\end{abstract}

\section{Source of material}

Reagents and solvents used were of commercially available reagent quality. As described in our previous paper [1], 4$\left(\mathrm{NO}_{2}\right)\left(\mathrm{C}_{6} \mathrm{H}_{4}\right) \mathrm{COOH}(1 \mathrm{mmol}, 0.17 \mathrm{~g})$ and $\mathrm{Ag}_{2} \mathrm{O}(0.5 \mathrm{mmol}, 0.12 \mathrm{~g})$ were dissolved in $30 \%$ ammonium solution $(10 \mathrm{ml})$, diethylenetriamine (1 mmol, $103 \mathrm{mg}$ ) was added with stirring for $1 \mathrm{~h}$. Crystals were formed after the filtrate was stood still in air for two days. The colorless crystals were filtrated and washed with ammonium solution and water, respectively, and dried in air (yield 93\%). Elemental analysis: found - C, 38.21\%; H, 4.24\%; N, 12.39\%; calc. for $\mathrm{C}_{18} \mathrm{H}_{24} \mathrm{AgN}_{5} \mathrm{O} 9-\mathrm{C}, 38.45 \% ; \mathrm{H}, 4.30 \% ; \mathrm{N}, 12.46 \%$.

\section{Experimental details}

All the H-atoms were located from Fourier maps and were refined isotropically. They were constrained to ride on their parent atoms with $\mathrm{N}-\mathrm{H}$ and $\mathrm{C}-\mathrm{H}$ distances of $0.90 \AA$ and $0.96 \AA$, respectively.

\section{Discussion}

Recently, we systematically reported [1] the synthesis and property of diethylenetriaminesilver(I) complexes containing various counter anions. As a continuation, we prepared and structurally characterized the crystal structure of the title complex. One primary amine $\mathrm{N}$ atom in dien was protonized, so the protonized dien $\mathrm{H}^{+}$acted as a bidentate bridging ligand. The nice crystals of the title compound are not very stable and change to a white powder when kept in a vacuum desiccator at room temperature for more than one day. The elemental analysis data and IR spectrum showed that the white powder was the same as the compound reported previously in [1]. Reported in this paper is a polymeric diethylenetriammonium silver(I) complex.

In the structure of the title compound each simplest repeating unit consists of a diethylenetriammonium silver(I) dication, two 4-nitrobenzoate anions, and a lattice water molecule. In the cation, the central silver(I) atom is coordinated by two nitrogen atoms from different diethylenetriamine molecules at the distance of $\mathrm{Ag}(1)-\mathrm{N}(2)=2.171(2) \AA$ and $\mathrm{Ag}(1)-\mathrm{N}(1 \mathrm{~A})=2.150(2) \AA$. The $\mathrm{N}-\mathrm{Ag}-\mathrm{N}$ angle is close to $180^{\circ}\left(174.32(9)^{\circ}\right)$, indicating a slightly distorted environment of $\operatorname{Ag}(1)$. One of the three nitrogen atoms in the dien ligand is protonated, namely $\mathrm{N}(3)$. The dien acts as a bidentate ligands by $\mathrm{N}(1)$ and $\mathrm{N}(2)$ atoms ligating to different silver(I) atoms. The one-dimensional chain ${ }_{\infty}^{1}[\mathrm{Ag}($ dien $)]$ stretches along $a$-axis. The 4-nitrobenzoates locate at different sides of the 1D chain, and the two aromatic rings are nearly parallel to each other with the dihedral angle of $4.40(2)^{\circ}$. A great number of hydrogen bonds join the complex to form a three-dimensional network.

\footnotetext{
* Correspondence author (e-mail: hlzhu@wist.edu.cn)
} 
Table 1. Data collection and handling.

$\begin{array}{ll}\text { Crystal: } & \text { yellow block, size } 0.22 \times 0.26 \times 0.50 \mathrm{~mm} \\ \text { Wavelength: } & \text { Mo } K_{\alpha} \text { radiation }(0.71073 \AA) \\ \mu: & 9.85 \mathrm{~cm}^{-1} \\ \text { Diffractometer, scan mode: } & \text { Siemens SMART CCD, } \omega \\ 2 \theta_{\text {max }}: & 56.6^{\circ} \\ N(h k l)_{\text {measured }}, N(h k l)_{\text {unique }}: & 13779,5366 \\ \text { Criterion for } I_{\mathrm{obs}}, N(h k l)_{\mathrm{gt}}: & I_{\mathrm{obs}}>\sigma\left(I_{\mathrm{obs}}\right), 4632 \\ N(\text { param })_{\text {refined }} & 394 \\ \text { Programs: } & \text { SHELXTL [2], SHEXTL-plus [3] }\end{array}$

Table 2. Atomic coordinates and displacement parameters (in $\AA^{2}$ ).

\begin{tabular}{llllll}
\hline Atom & Site & $x$ & $y$ & $z$ & $U_{\text {iso }}$ \\
\hline $\mathrm{H}(1 \mathrm{~A})$ & $4 e$ & $0.707(4)$ & $0.218(1)$ & $0.321(2)$ & $0.032(7)$ \\
$\mathrm{H}(1 \mathrm{~B})$ & $4 e$ & $0.556(5)$ & $0.204(1)$ & $0.225(2)$ & $0.041(8)$ \\
$\mathrm{H}(2 \mathrm{~A})$ & $4 e$ & $0.792(5)$ & $0.132(1)$ & $0.344(2)$ & $0.041(8)$ \\
$\mathrm{H}(2 \mathrm{~B})$ & $4 e$ & $0.623(4)$ & $0.122(1)$ & $0.249(2)$ & $0.033(7)$
\end{tabular}

Table 2. Continued.

\begin{tabular}{llllll}
\hline Atom & Site & $x$ & $y$ & \multicolumn{1}{l}{$z$} & $U_{\text {iso }}$ \\
\hline $\mathrm{H}(3 \mathrm{~A})$ & $4 e$ & $0.599(5)$ & $0.082(1)$ & $0.450(2)$ & $0.043(8)$ \\
$\mathrm{H}(3 \mathrm{~B})$ & $4 e$ & $0.436(5)$ & $0.069(1)$ & $0.358(3)$ & $0.049(9)$ \\
$\mathrm{H}(4 \mathrm{~A})$ & $4 e$ & $0.301(6)$ & $0.118(1)$ & $0.527(3)$ & $0.06(1)$ \\
$\mathrm{H}(4 \mathrm{~B})$ & $4 e$ & $0.142(7)$ & $0.100(2)$ & $0.433(3)$ & $0.08(1)$ \\
$\mathrm{H}(6)$ & $4 e$ & $-0.667(5)$ & $0.151(1)$ & $-0.209(2)$ & $0.035(7)$ \\
$\mathrm{H}(7)$ & $4 e$ & $-0.933(5)$ & $0.159(1)$ & $-0.349(2)$ & $0.036(7)$ \\
$\mathrm{H}(9)$ & $4 e$ & $-1.346(5)$ & $0.220(1)$ & $-0.185(2)$ & $0.036(7)$ \\
$\mathrm{H}(10)$ & $4 e$ & $-1.089(5)$ & $0.214(1)$ & $-0.035(2)$ & $0.050(9)$ \\
$\mathrm{H}(13)$ & $4 e$ & $0.661(6)$ & $0.077(1)$ & $0.851(3)$ & $0.06(1)$ \\
$\mathrm{H}(14)$ & $4 e$ & $0.821(5)$ & $0.087(1)$ & $1.015(2)$ & $0.044(8)$ \\
$\mathrm{H}(16)$ & $4 e$ & $1.368(6)$ & $0.030(1)$ & $0.947(3)$ & $0.06(1)$ \\
$\mathrm{H}(17)$ & $4 e$ & $1.207(5)$ & $0.019(1)$ & $0.779(2)$ & $0.040(8)$ \\
$\mathrm{H}(1 \mathrm{~N} 1)$ & $4 e$ & $0.879(5)$ & $0.227(1)$ & $0.184(2)$ & $0.045(9)$ \\
$\mathrm{H}(2 \mathrm{~N} 1)$ & $4 e$ & $0.823(6)$ & $0.181(1)$ & $0.151(3)$ & $0.05(1)$ \\
$\mathrm{H}(1 \mathrm{~N} 2)$ & $4 e$ & $0.509(5)$ & $0.158(1)$ & $0.407(2)$ & $0.037(9)$ \\
$\mathrm{H}(1 \mathrm{~N} 3)$ & $4 e$ & $0.395(8)$ & $0.039(2)$ & $0.573(4)$ & $0.10(2)$ \\
$\mathrm{H}(2 \mathrm{~N} 3)$ & $4 e$ & $0.136(6)$ & $0.043(1)$ & $0.557(3)$ & $0.06(1)$ \\
$\mathrm{H}(3 \mathrm{~N} 3)$ & $4 e$ & $0.227(5)$ & $0.018(1)$ & $0.474(2)$ & $0.037(8)$ \\
$\mathrm{H}(1 \mathrm{~W} 1)$ & $4 e$ & $0.002(6)$ & $0.206(1)$ & $0.494(3)$ & $0.05(1)$ \\
& & & & &
\end{tabular}

Table 3. Atomic coordinates and displacement parameters (in $\AA^{2}$ ).

\begin{tabular}{|c|c|c|c|c|c|c|c|c|c|c|}
\hline Atom & Site & $x$ & $y$ & $z$ & $U_{11}$ & $U_{22}$ & $U_{33}$ & $U_{12}$ & $U_{13}$ & $U_{23}$ \\
\hline $\operatorname{Ag}(1)$ & $4 e$ & $0.16889(3)$ & $0.172472(8)$ & $0.28610(2)$ & $0.0226(1)$ & $0.0489(1)$ & $0.0464(1)$ & $0.00378(8)$ & $0.00699(8)$ & $0.01326(9)$ \\
\hline $\mathrm{O}(1)$ & $4 e$ & $-0.7493(5)$ & $0.1867(1)$ & $0.0661(2)$ & $0.070(2)$ & $0.128(2)$ & $0.029(1)$ & $0.015(2)$ & $0.001(1)$ & $0.006(1)$ \\
\hline $\mathrm{O}(2)$ & $4 e$ & $-0.5175(4)$ & $0.1578(1)$ & $-0.0215(2)$ & $0.043(1)$ & $0.081(2)$ & $0.056(1)$ & $0.008(1)$ & $-0.006(1)$ & $0.006(1)$ \\
\hline $\mathrm{O}(3)$ & $4 e$ & $-1.2707(3)$ & $0.18591(9)$ & $-0.4645(2)$ & $0.035(1)$ & $0.093(2)$ & $0.031(1)$ & $-0.005(1)$ & $0.0035(8)$ & $-0.015(1)$ \\
\hline $\mathrm{O}(4)$ & $4 e$ & $-1.5257(3)$ & $0.20515(8)$ & $-0.3715(2)$ & $0.0274(9)$ & $0.064(1)$ & $0.041(1)$ & $0.0008(9)$ & $0.0021(8)$ & $-0.0075(9)$ \\
\hline $\mathrm{O}(5)$ & $4 e$ & $0.9035(4)$ & $0.02770(9)$ & $0.6206(2)$ & $0.051(1)$ & $0.081(2)$ & $0.039(1)$ & $-0.021(1)$ & $0.0131(9)$ & $-0.013(1)$ \\
\hline $\mathrm{O}(7)$ & $4 e$ & $1.1134(6)$ & $0.0782(1)$ & $1.1678(2)$ & $0.152(3)$ & $0.075(2)$ & $0.042(1)$ & $-0.013(2)$ & $-0.002(2)$ & $-0.016(1)$ \\
\hline $\mathrm{O}(8)$ & $4 e$ & $1.4171(6)$ & $0.0526(1)$ & $1.1263(2)$ & $0.103(2)$ & $0.086(2)$ & $0.071(2)$ & $-0.006(2)$ & $-0.047(2)$ & $0.000(2)$ \\
\hline $\mathrm{N}(1)$ & $4 e$ & $0.8597(3)$ & $0.1963(1)$ & $0.2022(2)$ & $0.029(1)$ & $0.042(1)$ & $0.030(1)$ & $-0.0041(9)$ & $0.0045(9)$ & $0.004(1)$ \\
\hline $\mathrm{N}(2)$ & $4 e$ & $0.4753(3)$ & $0.14141(8)$ & $0.3629(2)$ & $0.025(1)$ & $0.034(1)$ & $0.029(1)$ & $-0.0029(8)$ & $0.0045(8)$ & $-0.0017(9)$ \\
\hline $\mathrm{N}(3)$ & $4 e$ & $0.2597(4)$ & $0.04386(9)$ & $0.5234(2)$ & $0.032(1)$ & $0.046(1)$ & $0.032(1)$ & $-0.006(1)$ & $0.0045(9)$ & $0.004(1)$ \\
\hline $\mathrm{N}(4)$ & $4 e$ & $-0.6953(4)$ & $0.17509(9)$ & $-0.0152(2)$ & $0.042(1)$ & $0.046(1)$ & $0.037(1)$ & $-0.006(1)$ & $-0.000(1)$ & $0.011(1)$ \\
\hline $\mathrm{N}(5)$ & $4 e$ & $1.2240(7)$ & $0.0639(1)$ & $1.1052(2)$ & $0.105(3)$ & $0.039(2)$ & $0.046(2)$ & $-0.019(2)$ & $-0.019(2)$ & $0.002(1)$ \\
\hline $\mathrm{C}(1)$ & $4 e$ & $0.6802(4)$ & $0.1947(1)$ & $0.2621(2)$ & $0.022(1)$ & $0.037(1)$ & $0.034(1)$ & $0.003(1)$ & $0.005(1)$ & $0.003(1)$ \\
\hline$C(3)$ & $4 e$ & $0.4552(4)$ & $0.0919(1)$ & $0.4076(2)$ & $0.030(1)$ & $0.038(1)$ & $0.036(1)$ & $-0.001(1)$ & $0.005(1)$ & $0.004(1)$ \\
\hline $\mathrm{C}(4)$ & $4 e$ & $0.2739(5)$ & $0.0922(1)$ & $0.4718(3)$ & $0.034(2)$ & $0.046(2)$ & $0.053(2)$ & $0.002(1)$ & $0.013(1)$ & $0.013(1)$ \\
\hline $\mathrm{C}(5)$ & $4 e$ & $-0.8580(4)$ & $0.18085(9)$ & $-0.1093(2)$ & $0.034(1)$ & $0.033(1)$ & $0.030(1)$ & $-0.005(1)$ & $0.003(1)$ & $0.007(1)$ \\
\hline$C(6)$ & $4 e$ & $-0.8065(4)$ & $0.1642(1)$ & $-0.2011(2)$ & $0.029(1)$ & $0.042(2)$ & $0.037(1)$ & $0.002(1)$ & $0.008(1)$ & $0.003(1)$ \\
\hline$C(7)$ & $4 e$ & $-0.9631(4)$ & $0.1688(1)$ & $-0.2885(2)$ & $0.035(1)$ & $0.041(1)$ & $0.030(1)$ & $0.001(1)$ & $0.009(1)$ & $-0.004(1)$ \\
\hline $\mathrm{C}(8)$ & $4 e$ & $-1.1661(4)$ & $0.18926(9)$ & $-0.2842(2)$ & $0.031(1)$ & $0.031(1)$ & $0.029(1)$ & $-0.0062(9)$ & $0.0062(9)$ & $-0.0022(9)$ \\
\hline $\mathrm{C}(9)$ & $4 e$ & $-1.2111(4)$ & $0.20565(9)$ & $-0.1895(2)$ & $0.031(1)$ & $0.036(1)$ & $0.032(1)$ & $0.001(1)$ & $0.009(1)$ & $-0.002(1)$ \\
\hline$C(10)$ & $4 e$ & $-1.0572(4)$ & $0.2021(1)$ & $-0.1017(2)$ & $0.042(2)$ & $0.039(1)$ & $0.026(1)$ & $-0.000(1)$ & $0.008(1)$ & $0.000(1)$ \\
\hline $\mathrm{C}(11)$ & $4 e$ & $-1.3350(4)$ & $0.1940(1)$ & $-0.3810(2)$ & $0.029(1)$ & $0.038(1)$ & $0.031(1)$ & $-0.007(1)$ & $0.004(1)$ & $-0.005(1)$ \\
\hline$C(12)$ & $4 e$ & $0.9139(4)$ & $0.04744(9)$ & $0.7973(2)$ & $0.032(1)$ & $0.036(1)$ & $0.032(1)$ & $-0.007(1)$ & $0.002(1)$ & $0.002(1)$ \\
\hline $\mathrm{C}(13)$ & $4 e$ & $0.8015(5)$ & $0.0677(1)$ & $0.8698(2)$ & $0.040(2)$ & $0.043(2)$ & $0.043(2)$ & $0.001(1)$ & $0.006(1)$ & $0.002(1)$ \\
\hline $\mathrm{C}(14)$ & $4 e$ & $0.9021(6)$ & $0.0736(1)$ & $0.9708(2)$ & $0.064(2)$ & $0.041(2)$ & $0.037(2)$ & $-0.002(1)$ & $0.010(1)$ & $-0.007(1)$ \\
\hline$C(15)$ & $4 e$ & $1.1158(5)$ & $0.0595(1)$ & $0.9963(2)$ & $0.062(2)$ & $0.031(1)$ & $0.034(1)$ & $-0.011(1)$ & $-0.010(1)$ & $0.002(1)$ \\
\hline$C(16)$ & $4 e$ & $1.2328(5)$ & $0.0401(1)$ & $0.9263(2)$ & $0.038(2)$ & $0.047(2)$ & $0.050(2)$ & $-0.002(1)$ & $-0.010(1)$ & $0.009(1)$ \\
\hline $\mathrm{C}(17)$ & $4 e$ & $1.1303(5)$ & $0.0344(1)$ & $0.8260(2)$ & $0.038(2)$ & $0.043(2)$ & $0.043(2)$ & $-0.001(1)$ & $0.004(1)$ & $-0.002(1)$ \\
\hline $\mathrm{C}(18)$ & $4 e$ & $0.7938(5)$ & $0.0386(1)$ & $0.6892(2)$ & $0.041(2)$ & $0.051(2)$ & $0.032(1)$ & $-0.011(1)$ & $0.001(1)$ & $0.003(1)$ \\
\hline
\end{tabular}

Acknowledgments. The authors thank the Education Office of Hubei Province P. R. China (grant No. 2002B29002) and the Natural Science Foundation of Hubei Province P. R. China (grant No. 2003ABB010).

\section{References}

1. Zhu, H.-L.; Wang, X.-J.; Liu X.-Y.; Wang, D.-Q.: Synthesis and Characterization of $\mathrm{Ag}(\mathrm{I})$ Salts with Diethylenetriamine. Crystal structure of $\left[\mathrm{Ag}_{2}(\text { dien })_{2}\right]\left(\mathrm{ClO}_{4}\right)_{2}$. Synth. React. Inorg., Met-Org. Chem. 33 (2003) $1417-1424$.
2. Sheldrick, G. M.: Siemens SHELXTL (Version 5.0): Siemens Industrial Automation, Inc., analytical Instrumentation, USA 1995.

3. Sheldrick, G. M.: SHELXTL-plus. Release 4.1. Siemens Analytial X-ray Instruments Inc., Madison Wisconsin, USA 1991. 